\title{
Vibrational spectrum and molecular structure of triphenylamine monomer: A combined matrix-isolation FTIR and theoretical study $\dagger$
}

\author{
Igor Reva, ${ }^{* a}$ Leszek Lapinski, ${ }^{a b}$ Nitin Chattopadhyay ${ }^{c}$ and Rui Fausto ${ }^{a}$ \\ a Departamento de Química, Universidade de Coimbra, 3004-535 Coimbra, Portugal. \\ E-mail: reva@qui.uc.pt \\ ${ }^{b}$ Institute of Physics, Polish Academy of Sciences, Al. Lotnikow 32/46, 02-668 Warsaw, Poland \\ ${ }^{c}$ Department of Chemistry, Jadavpur University, Calcutta 700 032, India
}

Received 10th June 2003, Accepted 8th July 2003

First published as an Advance Article on the web 6th August 2003

Theoretical optimization of triphenylamine geometry, carried out at DFT(B3LYP) level using 6-31G** and aug-cc-pVDZ basis sets, predicted a propeller-like structure of the compound with $D_{3}$ overall symmetry. In this structure, the central NCCC atoms are coplanar and the phenyl rings are symmetrically twisted from this plane by $41.5^{\circ}\left(6-31 \mathrm{G}^{* *}\right)$ or $41.6^{\circ}$ (aug-cc-pVDZ). The experimental FTIR spectrum of triphenylamine monomers isolated in an argon matrix was measured and interpreted by comparison with theoretical spectra calculated at the DFT(B3LYP) level with 6-31G** or aug-cc-pVDZ basis sets. The good agreement between the experimental and theoretical spectra allowed a positive assignment of the observed infrared absorption bands.

Conformational flexibility of triphenylamine was investigated by carrying out a series of theoretical scans of the potential energy hypersurface of the system. Special attention was granted to the minimal energy pathway between the left-hand rotating and right-hand rotating symmetry identical structures of the compound. A route conserving a $C_{2}$ symmetry axis was identified as implying an energy barrier of $20 \mathrm{~kJ} \mathrm{~mol}^{-1}$ only, whereas the calculated barrier for the concerted twist of all the phenyl rings (the route with conservation of the $C_{3}$ symmetry axis) was as high as $54 \mathrm{~kJ} \mathrm{~mol}^{-1}$.

\section{Introduction}

Because of their huge potential in diverse fields, triphenylamine (TPA) and several of its derivatives have drawn the attention of the photoscientists since long. These compounds have received application in organic light emitting diodes (OLED) (particularly in the blue region), ${ }^{1-6}$ as photoconductors ${ }^{7-9}$ and semiconductors, ${ }^{10}$ as laser dyes, ${ }^{11,12}$ in photodiodes ${ }^{13,14}$ and as mechanoluminescent ${ }^{15}$ or triboluminescent ${ }^{16}$ materials. The dendrimer formation from TPA-based molecular systems ${ }^{17,18}$ and their photocyclization to form carbazole derivatives $^{19}$ have also been the subject of intense research. Both their geometric ${ }^{20-28}$ and electronic ${ }^{29}$ structural characteristics have been addressed by several authors.

Previous experimental ${ }^{24-28,30}$ as well as theoretical ${ }^{20-22}$ studies on the structure of TPA suggest a three-bladed propeller structure for the molecule (Fig. 1) with a planar or nearly planar central NCCC moiety. In the study of high resolution laser induced fluorescence (LIF) excitation spectra of TPA in a supersonic jet expansion, ${ }^{25}$ a symmetric top geometry, with at least $C_{3}$ symmetry, was experimentally established for the molecule in the gas phase. The rotationally resolved experimental LIF excitation spectra could be best reproduced by a theoretical fit when the $\mathrm{CNC}$ angle was assumed to be equal (or nearly equal) to $120^{\circ}$, and the angles between the plane

\footnotetext{
$\dagger$ Electronic supplementary information (ESI) available: Fig. S1 provides atom numbering and Table S1 optimized geometry parameters and rotational constants for the TPA molecule. Table $\mathrm{S} 2$ provides a list of calculated frequencies and descriptions for fully symmetrical $\left(\mathrm{A}_{1}\right)$ vibrations in the TPA monomer. Figs. S2-S4 present changes in the TPA molecule geometry during potential energy scans with different symmetry restrictions.

See http://www.rsc.org/suppdata/cp/b3/b306489a/
}

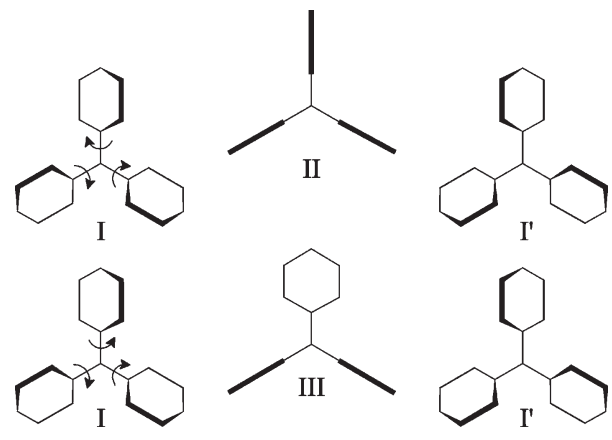

Fig. 1 Possible pathways for transformation of TPA from the minimum energy structure $\mathbf{I}$ to its mirror-like counterpart $\mathbf{I}^{\prime}$.

of the central NCCC moiety and those of the phenyl rings equal to $50^{\circ}$. In the solid state, TPA forms crystals with four non-equivalent molecules per unit cell. ${ }^{27}$ The average (over these four molecules) value of the $\mathrm{CNC}$ angle was determined as equal to $119.6^{\circ}$, whereas the mean value of the dihedral angle between the planes of the central NCCC atoms and of a phenyl ring was found to be $44^{\circ}$. Based on the analysis of the low frequency fragments of the infrared and Raman spectra of TPA dissolved in $\mathrm{CS}_{2}$, Rodionov et al. ${ }^{26}$ also proposed that the central NCCC skeleton is planar. On the other hand, TPA was found to exhibit a non-vanishing dipole moment in diluted solutions. ${ }^{28,31}$ This is consistent with a molecular geometry showing a slight non-planarity at the central nitrogen atom.

Although the previously reported structural studies on TPA in general agree that the molecule should adopt the propeller shape with exact or approximate $D_{3}$ symmetry, little is known about the conformational flexibility of the system. In addition, 
the complete mid-infrared spectrum of TPA has never been reported and interpreted.

In the current paper, the FTIR spectrum of TPA monomers isolated in a low-temperature argon matrix is reported and compared with the theoretical spectrum calculated at the DFT(B3LYP) level of theory. This comparison enabled us to carry out an extensive assignment of experimental spectrum and did also provide fundamental information for structural characterization of the studied molecule. In order to look at the conformational flexibility of TPA, the vibrational studies were complemented by a detailed theoretical investigation of the potential energy hypersurface of the molecule. Two pathways for interconversion between the two mirror-like potential energy minima of the molecule were considered. It was shown that not the straightforward way with three phenyl rings rotating in the same direction, but a channel where two rings rotate in the same direction and the third in the opposite should be preferred. This result provides an interesting and non-trivial example of conformational behaviour of a complex molecule.

\section{Experimental}

The sample of triphenylamine (TPA) used in the present study was purchased from Aldrich. It was purified by recrystallization followed by vacuum sublimation prior to the matrix experiments. To deposit a matrix, the compound was sublimated at $323 \mathrm{~K}$ from a glass mini oven placed in the vacuum chamber of the cryostat. The vapours of the compound were deposited, together with a large excess of argon N60 (Air Liquide), onto a CsI window mounted on the cold (9 K) finger of an APD Cryogenics closed-cycle helium refrigerator with a DE-202A expander.

The IR spectra $\left(0.5 \mathrm{~cm}^{-1}\right.$ resolution) were recorded in the range $4000-400 \mathrm{~cm}^{-1}$ using a Mattson (Infinity 60AR Series) Fourier-transform infrared spectrometer with a DTGS detector and a $\mathrm{Ge} / \mathrm{KBr}$ beamsplitter. The spectrometer was blown with dry air in order to exclude influence of atmospheric moisture and $\mathrm{CO}_{2}$.

\section{Computational details}

Geometry optimizations were carried out at the DFT level with the three-parameter B3LYP functional that includes Becke's gradient exchange correction, ${ }^{32}$ the Lee, Yang, Parr correlation functional ${ }^{33}$ and the Vosko, Wilk and Nusair ${ }^{34}$ correlation functional. The standard $6-31 \mathrm{G}^{* *}$ and aug-ccpVDZ basis sets were used in these calculations.

The geometry optimizations were followed by frequency calculations to check the nature of the located stationary points. The calculated frequencies were used to assist the analysis of the experimental spectra.

All calculations were done using the Gaussian 98 program. ${ }^{35}$ The GaussView 2.1 program (Semichem, Inc.) was used for the visualization of the calculated vibrational modes of TPA.

\section{Results and discussion}

\section{Minimum-energy structure}

The geometry of TPA was optimized at the DFT(B3LYP)/6$31 \mathrm{G}^{* *}$ and DFT(B3LYP)/aug-cc-pVDZ levels of theory. Full geometry optimization led to a single minimum energy structure I, with $D_{3}$ symmetry, where the three phenyl groups are symmetrically equivalent (Fig. 1). In this structure, the central nitrogen and the three adjacent carbon atoms are co-planar, the planes of the three phenyl substituents being symmetrically twisted from the plane of the central NCCC moiety. Consistent with previously reported experimental data, ${ }^{27}$ the molecule is predicted by the present calculations to have a propeller-like geometry, the phenyl substituents corresponding to the propeller blades.

The minimum-energy structure of TPA, with the phenyl rings twisted with respect to the plane of the central NCCC moiety, can be rationalized in terms of the interplay between two opposing factors: $(i)$ the energy-lowering conjugation of the $\pi$-electron systems of the phenyl groups and (ii) the energy-increasing repulsion between the protons of neighbouring phenyl rings. The conjugation of the $\pi$-electron systems of the three phenyl rings would be maximized in the totally planar conformation (with $D_{3 \mathrm{~h}}$ symmetry). However, for such geometry, the steric repulsion between the inter-ring hydrogen atoms, placed at very short distance from each other, would be very pronounced. On the other hand, a conformation with the three phenyl groups perpendicular to the plane of the central NCCC moiety would lead to a minimal repulsion between the hydrogen atoms, but the conjugation of the $\pi$-electron systems would be lost.

The minimum energy conformer I of TPA has a symmetry identical counterpart $\mathbf{I}^{\prime}$, which is its mirror image (Fig. 1). The possible transition pathways from one mirror form to the other will be discussed in detail in a forthcoming section.

The structural parameters, optimized for TPA in the present work, are listed in Table $\mathrm{S} 1$ and the atom numbering scheme adopted in this study is presented in the electronic supplementary information (ESI, $\uparrow$ Fig. S1). As it can be seen from this Table, the geometries (bond lengths and angles) calculated using both the DFT(B3LYP)/6-31G** and DFT(B3LYP)/ aug-cc-pVDZ levels of theory are practically equal. An important geometrical parameter in the molecule of TPA is the angle between the central NCCC planar moiety and the planes of the phenyl rings. This angle can be defined as CNCC torsion. From here onwards we shall call this angle "pitch of the blade". For a totally planar TPA molecule, the pitch of the blade would be equal to $0^{\circ}$. In the aug-cc-pVDZ optimized geometry, the pitch of the blade was found to be $41.6^{\circ}\left(41.5^{\circ}\right.$ at the $6-31 \mathrm{G}^{* *}$ level). It is in good agreement with the available experimental data: $c a$. $50^{\circ}$ for TPA in the gaseous phase ${ }^{25}$ and $44^{\circ}$ in crystalline TPA-average value for this parameter in the non equivalent molecules of the crystal unit cell. ${ }^{27}$

Summarizing this section let us note that the theoretical calculations, performed without any restriction of symmetry, revealed that the central NCCC fragment of TPA molecule at the monomer level is planar. The molecule has overall $D_{3}$ symmetry and its total dipole moment is equal to zero. The analysis of the vibrational spectrum gives further confirmation that the geometry reported in Table S1 corresponds to the true minimum on the potential energy surface of TPA.

\section{Matrix isolation FTIR studies}

The experimental infrared spectrum of TPA was recorded for the monomers of the compound isolated in an argon matrix. This spectrum is presented in Fig. 2. The frequencies of the observed bands and their integral intensities are listed in Table 1. The experimental spectrum (Fig. 2, upper frame) is compared with the spectra calculated using density functional theory at the 6-31G** (lower frame) and aug-cc-pVDZ (middle frame) levels, obtained under the harmonic approximation, for the minimum-energy $D_{3}$ symmetry structure. The general agreement between the experimental and the calculated spectra is good, suggesting a close match between the theoretically optimized geometry of TPA and the structure of the molecule in the matrix.

In spite of the good agreement between the experimental and calculated spectra, as usual the theoretical frequencies are slightly overestimated, mainly due to neglect of anharmonicity. In the fingerprint region $\left(1600-400 \mathrm{~cm}^{-1}\right)$ the frequency ratio (experimental/calculated) varies from 0.992 to 0.966 


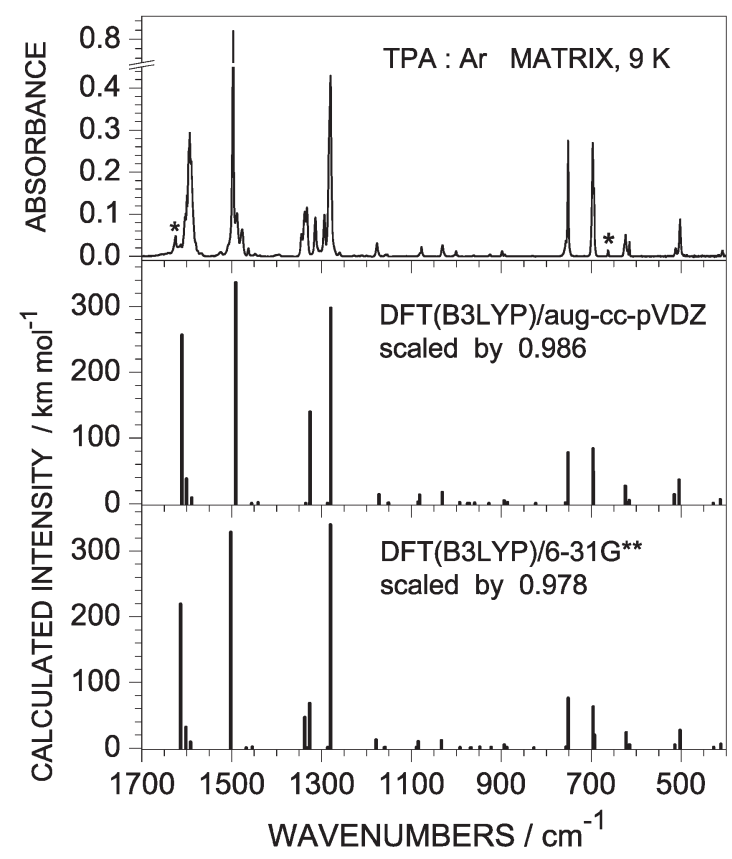

Fig. 2 Comparison of the FTIR spectrum of triphenylamine isolated in an argon matrix at $9 \mathrm{~K}$ with the spectra calculated at DFT(B3LYP)/ 6-31G** and DFT(B3LYP)/aug-cc-pVDZ levels of theory for monomer. The theoretical spectra are scaled by 0.978 and 0.986 respectively. The choice of the scaling factors is discussed in text. Asterisks in the experimental spectrum designate bands due to traces of $\mathrm{CO}_{2}$ and $\mathrm{H}_{2} \mathrm{O}$ in the matrix.

and from 0.995 to 0.975 , for the calculations with the $6-31 \mathrm{G}^{* *}$ and the aug-cc-pVDZ basis set respectively, the corresponding average ratios being 0.978 and 0.986 . Using these scaling factors, the average deviations of the scaled theoretical frequencies from the experimentally observed ones reduce to 4.5 and $4.1 \mathrm{~cm}^{-1}$, for the $6-31 \mathrm{G}^{* *}$ and aug-cc-pVDZ basis sets, respectively. Hence, while a priori the aug-cc-pVDZ calculations predict the frequencies closer (by $1 \%$ ) to experiment than those performed with the $6-31 \mathrm{G}^{*}$ basis set, the subsequent use of the above scaling factors enabled to attain a quantitatively identical prediction of the experimental spectrum with both basis sets. In practical terms, this means that the $6-31 \mathrm{G}^{* *}$ basis set (which requires a substantially smaller computational effort) can be used instead of the more extended aug-cc-pVDZ basis to further investigate TPA without any significant loss of quality in the theoretical predictions.

The normal modes of TPA can be divided into two groups: those involving predominantly the NCCC moiety and those associated with the phenyl rings. The classification of the normal modes of monosubstituted benzene derivatives is given in the work of Whiffen. ${ }^{36}$ The vibrations performed by the phenyl groups of TPA follow this classification nicely. In a symmetric molecule with three phenyl groups, each type of vibration described by Whiffen must be symmetry coupled with the corresponding vibrations in the other two units. As it is described below, this fact is of importance for examination of TPA conformational flexibility and dynamics.

The interpretation of the infrared spectrum of TPA isolated in Ar matrix is given in Table 1. In this Table, the symbols introduced by Whiffen are used for description of phenyl normal modes. In a molecule with $D_{3}$ symmetry, the normal vibrations can be either fully symmetric (belonging to $A_{1}$ representation and inactive in infrared) or belong to the $A_{2}$ or $\mathrm{E}$ representations, active in infrared. For the last two representations, the frequencies and intensities of the theoretically predicted IR bands are listed in Table 1. The results of calculations of fully symmetric $A_{1}$ normal modes are given in Table S2 of the ESI. $\dagger$
The bands due to the $\mathrm{CH}$ stretching vibrations are observed as a group of partially overlapping absorptions in the region $3110-3015 \mathrm{~cm}^{-1}$. In the spectral range below $1700 \mathrm{~cm}^{-1}$, the strongest bands, observed at 1496.6 and $1280.2 \mathrm{~cm}^{-1}$, correspond to normal vibrations with significant contributions from the $\mathrm{CN}$ stretching coordinates. The strongest band due to vibrations of the phenyl rings is observed at $1593.3 \mathrm{~cm}^{-1}$. Intense bands at such frequency are usually observed in the IR spectra of monosubstituted benzene derivatives. ${ }^{37}$ Another band that is characteristic of compounds with phenyl groups is observed at $751.7 \mathrm{~cm}^{-1}$. It corresponds to the out-of-plane (wagging) vibration of the hydrogen atoms attached to the phenyl rings. Below $800 \mathrm{~cm}^{-1}$, the IR spectrum of TPA is dominated by the bands due to ring in-plane bending vibrations (bands at 623.8 and $619.3 \mathrm{~cm}^{-1}$ ) and by those corresponding to the out-of-plane (puckering) vibrations of the phenyl rings (bands at 696.9, 694.1, 512.5, 502.4 and 408.1 $\mathrm{cm}^{-1}$ ). Noteworthy, the bands due to the same type of local (phenyl) symmetry vibrations, which are coupled according to the $\mathrm{A}_{2}$ or E global symmetry patterns, are observed as closely separated doublets (see Fig. 2 and Table 1); in general one member of the doublet is intense and the other much weaker.

The lowest frequency vibrations theoretically predicted for TPA are performed along the coordinates corresponding to the shallowest cuts through the potential energy hypersurface of the molecule. Torsions of the phenyl moieties around the $\mathrm{CN}$ bonds (changing the pitch of the propeller blade) correspond to coordinates with these characteristics. The calculated frequency for the totally symmetric (all in-phase; $\mathrm{A}_{1}$ ) torsion of the three phenyl groups is predicted to be $79.7 \mathrm{~cm}^{-1}$ at the aug-cc-pVDZ level, (Table S2), while that corresponding to the degenerated (E-symmetry) phenyl torsional vibrations corresponds to the lowest predicted frequency, $35.4 \mathrm{~cm}^{-1}$ (Table 1). The vibration corresponding to pyramidalization at the central nitrogen atom $\left(\mathrm{A}_{2}\right.$ symmetry) was also predicted to have a very low frequency, $45.7 \mathrm{~cm}^{-1}$. Such low frequencies indicate that the change in the pitch of the blade and the pyramidalization at nitrogen atom are the preferred directions of conformational flexibility in the TPA molecule.

\section{Conformational flexibility and conformational interconversion pathways}

As mentioned above, the minimum energy $D_{3}$ structure $\mathbf{I}$ has a symmetry related identical counterpart $\mathbf{I}^{\prime}$, which is its mirror image (Fig. 1). In a good approximation, the three phenyl groups of TPA can be treated as planar, rigid units. Hence, the investigation of possible transition pathways converting one form to the other can be simplified to consideration of only two types of conformationally relevant parameters: rotations of the phenyl groups around the $\mathrm{CN}$ bonds and pyramidalization at the central nitrogen atom. In fact, the possibility of such simplification could be deduced from the nature of the normal modes corresponding to the lowest frequency vibrations in TPA.

Conversion from the left-hand to the right-hand rotating propeller geometry (and vice-versa) is possible by twisting all phenyl groups around $\mathrm{CN}$ bonds in the same direction. However, this is not the only conceivable way for interconversion between the symmetrically identical minimum energy TPA structures: this conversion can also be achieved by twisting two phenyls in one direction and the third in the opposite way. The two possible pathways are presented in Fig. 1.

In order to evaluate the barrier for the conversion from one TPA minimum to its symmetrically identical counterpart, a series of systematic theoretical [DFT(B3LYP)/6-31G**] potential energy scans was carried out. The reaction coordinates were chosen with symmetry of the system taken into account. The minimum-energy geometry of TPA belongs to the $D_{3}$ symmetry group, which includes the $C_{3}, C_{2}$ and $C_{1}$ 
Table 1 Experimental and calculated frequencies $\left(\mathrm{cm}^{-1}\right)$ and IR intensities (in parentheses, $\mathrm{km} \mathrm{mol}^{-1}$ ) of the vibrations active in the IR spectrum of TPA monomer. Theoretical intensities for the doubly degenerate E symmetry modes are summarized. Theoretical frequencies calculated with the $6-31 \mathrm{G}^{* *}$ basis set are scaled with a factor equal to 0.978 , and those calculated with the aug-cc-pVDZ basis set scaled with a factor equal to 0.986 . m-merged bands; sh-shoulders. R-ring, str-stretching, bend-bending, oop-out-of-plane, tors-torsion

\begin{tabular}{|c|c|c|c|c|c|c|}
\hline \multirow{3}{*}{$\begin{array}{l}\text { Experimental frequency and } \\
\text { (relative integrated intensity) }^{a} \\
3107.0 \mathrm{~m}\end{array}$} & \multicolumn{4}{|c|}{ Calculated DFT(B3LYP) } & \multirow{3}{*}{$\begin{array}{l}\text { Symmetry } \\
\text { E }\end{array}$} & \multirow{3}{*}{$\begin{array}{l}\text { Description and Whiffen symbol } \\
\mathrm{CH} \text { str }\end{array}$} \\
\hline & \multicolumn{2}{|c|}{$6-31 G^{* *}$} & \multicolumn{2}{|c|}{ aug-cc-pVDZ } & & \\
\hline & 3143.7 & $(5.8)$ & 3159.4 & $(7.0)$ & & \\
\hline $3095.9 \mathrm{~m}$ & 3141.2 & $(21.0)$ & 3156.6 & $(18.5)$ & $\mathrm{E}$ & $\mathrm{CH}$ str \\
\hline $3067.0 \mathrm{~m}(131.1)$ & 3140.7 & $(18.5)$ & 3155.7 & $(11.2)$ & $\mathrm{A}_{2}$ & $\mathrm{CH}$ str \\
\hline $3043.4 \mathrm{~m}$ & 3132.9 & $(96.6)$ & 3149.8 & $(66.5)$ & $\mathrm{E}$ & $\mathrm{CH}$ str \\
\hline $3016.0 \mathrm{~m}$ & 3117.7 & $(20.9)$ & 3135.1 & $(18.6)$ & $\mathrm{E}$ & $\mathrm{CH}$ str \\
\hline & 3117.6 & $(18.0)$ & 3134.6 & $(10.0)$ & $\mathrm{A}_{2}$ & $\mathrm{CH}$ str \\
\hline & 3110.0 & $(12.0)$ & 3127.4 & $(10.8)$ & $\mathrm{E}$ & $\mathrm{CH}$ str \\
\hline \multicolumn{7}{|l|}{$1612.9 \mathrm{~m}$} \\
\hline \multicolumn{7}{|l|}{$1602.3 \mathrm{~m}$} \\
\hline \multicolumn{7}{|l|}{$1599.6 \mathrm{~m}$} \\
\hline \multicolumn{7}{|l|}{$1595.3 \mathrm{~m}$} \\
\hline $1593.3 \mathrm{~m}(402.8)$ & 1613.7 & $(219.0)$ & 1610.4 & $(256.2)$ & $\mathrm{E}$ & $\mathrm{R} \operatorname{str}(\mathrm{k})$ \\
\hline \multirow[t]{2}{*}{$1589.8 \mathrm{~m}$} & 1601.9 & $(31.5)$ & 1600.5 & $(37.5)$ & $\mathrm{E}$ & $\mathrm{R} \operatorname{str}(1)$ \\
\hline & 1591.6 & $(8.9)$ & 1588.8 & $(8.5)$ & $\mathrm{A}_{2}$ & $\mathrm{R} \operatorname{str}(1)$ \\
\hline \multicolumn{7}{|l|}{$1507.3 \mathrm{~m}$} \\
\hline 1496.6 m (428.6) & 1502.1 & $(328.3)$ & 1490.9 & $(336.2)$ & $\mathrm{E}$ & $\mathrm{CN}$ str, R str (m), $\mathrm{CH}$ bend (b) \\
\hline \multicolumn{7}{|l|}{$1488.2 \mathrm{~m}$} \\
\hline \multicolumn{7}{|l|}{$1476.6 \mathrm{~m}$} \\
\hline $1462.7(6.0)$ & 1467.8 & $(0.1)$ & 1455.6 & $(0.1)$ & $\mathrm{E}$ & $\mathrm{CH}$ bend (d), R str (n) \\
\hline $1447.8(2.2)$ & 1454.1 & $(1.5)$ & 1441.2 & $(1.3)$ & $\mathrm{A}_{2}$ & $\mathrm{CH}$ bend (d), R str (n) \\
\hline $1344.7 \mathrm{~m}$ & & & & & & \\
\hline $1337.2 \mathrm{~m}(131.7)$ & 1337.5 & $(46.4)$ & 1335.7 & $(0.3)$ & $\mathrm{E}$ & $\mathrm{CH}$ bend (e), R str (o) \\
\hline & 1331.6 & $(0.1)$ & 1326.5 & $(0.7)$ & $\mathrm{A}_{2}$ & $\mathrm{CH}$ bend (e), R str (o) \\
\hline $1332.6 \mathrm{~m}$ & 1326.7 & $(67.9)$ & 1325.8 & $(139.2)$ & $\mathrm{E}$ & $\mathrm{CN}$ str, $\mathrm{CNC}$ bend, R str (o) \\
\hline & 1286.5 & $(0.8)$ & 1286.7 & $(0.5)$ & $\mathrm{A}_{2}$ & R str (o) \\
\hline $1280.2(291.7)$ & 1280.5 & (239.6) & 1279.4 & $(297.1)$ & $\mathrm{E}$ & $\mathrm{CN}$ str \\
\hline $1177.0(16.1)$ & 1178.4 & (12.3) & 1172.2 & (13.8) & $\mathrm{E}$ & $\mathrm{CH}$ bend (a) \\
\hline $1157.1(2.2)$ & 1160.3 & $(0.5)$ & 1152.7 & $(0.4)$ & $\mathrm{E}$ & $\mathrm{CH}$ bend (c) \\
\hline $1153.3(0.4)$ & 1158.7 & $(1.0)$ & 1151.1 & $(0.6)$ & $\mathrm{A}_{2}$ & $\mathrm{CH}$ bend (c) \\
\hline $1081.2 \mathrm{~m}$ & 1088.6 & $(0.9)$ & 1085.6 & $(2.2)$ & $\mathrm{E}$ & $\mathrm{CH}$ bend $(\mathrm{d})$ \\
\hline 1077.6 m (9.9) & 1085.0 & $(9.5)$ & 1082.1 & $(12.8)$ & $\mathrm{A}_{2}$ & $\mathrm{CH}$ bend $(\mathrm{d})$ \\
\hline $1031.0(14.4)$ & 1033.6 & $(11.0)$ & 1031.7 & $(17.0)$ & $\mathrm{E}$ & $\mathrm{R} \operatorname{str}(\mathrm{m})$ \\
\hline $1005.9 \mathrm{~m}$ & & & & & & \\
\hline $1000.7 \mathrm{~m}(5.2)$ & 992.0 & $(0.8)$ & 993.0 & (1.6) & $\mathrm{E}$ & $\mathrm{R}$ bend (p) \\
\hline $997.8 \mathrm{~m}$ & & & & & & \\
\hline $976.5(0.3)$ & 968.5 & $(0.2)$ & 971.3 & $(0.1)$ & $\mathrm{E}$ & $\mathrm{CH}$ oop (j) \\
\hline & 967.5 & $(0.1)$ & 975.4 & $(0.1)$ & $\mathrm{A}_{2}$ & $\mathrm{CH}$ oop (j) \\
\hline $961.7(1.0)$ & 948.4 & $(1.5)$ & 959.8 & $(0.7)$ & $\mathrm{E}$ & $\mathrm{CH}$ oop (h) \\
\hline $925.4(1.7)$ & 923.1 & $(1.0)$ & 927.7 & $(0.1)$ & $\mathrm{E}$ & CNC bend, $\mathrm{R}$ bend (p), CHoop (i) \\
\hline $898.4(4.1)$ & 894.0 & $(4.8)$ & 893.9 & $(4.3)$ & $\mathrm{A}_{2}$ & $\mathrm{CH}$ oop (i) \\
\hline $892.7(1.5)$ & 887.7 & $(1.0)$ & 886.9 & $(1.4)$ & $\mathrm{E}$ & $\mathrm{CH}$ oop (i) \\
\hline $835.1 \mathrm{~m}$ & & & & & & \\
\hline $830.9 \mathrm{~m}(1.0)$ & 828.0 & $(0.2)$ & 823.7 & $(0.2)$ & $\mathrm{E}$ & $\mathrm{CH}$ oop (g) \\
\hline $756.7 \mathrm{~m}$ & 756.0 & $(1.5)$ & 757.4 & $(1.0)$ & $\mathrm{E}$ & $\mathrm{CH}$ oop (f) \\
\hline $751.7 \mathrm{~m}(103.7)$ & 751.6 & $(76.1)$ & 751.6 & $(77.6)$ & $\mathrm{A}_{2}$ & $\mathrm{CH}$ oop (f) \\
\hline $696.9 \mathrm{~m}(142.6)$ & 696.0 & $(62.8)$ & 696.3 & $(83.2)$ & $\mathrm{E}$ & $\mathrm{R}$ tors $(\mathrm{v}), \mathrm{CH}$ oop $(\mathrm{f})$ \\
\hline $694.1 \mathrm{~m}$ & 693.2 & $(19.8)$ & 695.3 & $(48.6)$ & $\mathrm{A}_{2}$ & $\mathrm{R}$ tors $(\mathrm{v}), \mathrm{CH}$ oop $(\mathrm{f})$ \\
\hline $626.3 \mathrm{sh}$ & & & & & & \\
\hline $623.8 \mathrm{~m}(29.1)$ & 622.4 & $(23.3)$ & 624.3 & $(26.4)$ & $\mathrm{E}$ & $\mathrm{R}$ bend $(\mathrm{t}), \mathrm{R}$ bend $(\mathrm{s})$ \\
\hline $619.3 \mathrm{~m}$ & 619.6 & $(0.7)$ & 620.5 & $(1.0)$ & $\mathrm{A}_{2}$ & $\mathrm{R}$ bend (s) \\
\hline $615.1(5.3)$ & 615.2 & $(5.0)$ & 615.7 & $(4.5)$ & $\mathrm{E}$ & $\mathrm{R}$ bend $(\mathrm{t}), \mathrm{R}$ bend $(\mathrm{s})$ \\
\hline $512.5(7.6)$ & 514.4 & $(4.8)$ & 515.4 & $(13.6)$ & $\mathrm{A}_{2}$ & $\mathrm{R}$ tors $(\mathrm{y})$ \\
\hline $502.4(36.7)$ & 502.7 & $(26.9)$ & 504.7 & (35.9) & $\mathrm{E}$ & $\mathrm{R}$ tors $(\mathrm{y})$ \\
\hline & 427.9 & $(1.0)$ & 428.9 & $(0.4)$ & $\mathrm{A}_{2}$ & $\mathrm{~N}$ out of CCC plane \\
\hline $408.1(5.4)$ & 411.7 & $(5.8)$ & 412.8 & $(6.1)$ & $\mathrm{E}$ & $\mathrm{R}$ tors $(\mathrm{w})$ \\
\hline & 327.0 & $(0.1)$ & 328.4 & $(0.2)$ & $\mathrm{E}$ & $\mathrm{Ph}$ bend $(\mathrm{u})$ \\
\hline & 238.3 & $(4.5)$ & 236.0 & $(4.7)$ & $\mathrm{E}$ & $\mathrm{R}$ tors $(\mathrm{x})$ \\
\hline & 208.8 & $(0.1)$ & 205.5 & $(0.2)$ & $\mathrm{A}_{2}$ & $\mathrm{Ph}$ bend $(\mathrm{u}), \mathrm{Ph}$ oop \\
\hline & 78.2 & $(0.0006)$ & 75.4 & $(0.001)$ & $\mathrm{E}$ & Phenyl wagging \\
\hline & 51.6 & $(0.5)$ & 45.7 & $(0.4)$ & $\mathrm{A}_{2}$ & Pyramidalization \\
\hline & 37.8 & $(4.0)$ & 35.4 & $(4.1)$ & $\mathrm{E}$ & Change of blade pitch \\
\hline
\end{tabular}

${ }^{a}$ List of observed bands due to overtones or combination tones. Peaks of merged bands are separated with a slash, relative intensities in parentheses: 2625.9/2616.3 (4.2); 2562.0 (4.8); 2008.9/1995.3 (2.4); 1949.9/1935.4/1923.5 (13.6); 1891.6 (1.0); 1867.5/1854.2 (7.8); 1791.3 (7.8); 1726.1 (6.8); 1523.9 (5.7); 1395.2 (4.9); 1313.8 (40.0); 1293.9 (40.4); 1259.8 (3.3); 1227.2 (1.1); 1217.2 (0.3); 1210.6 (1.1); $1199.6(0.9) ; 987.4$ (0.1); 1624.7 (14.2)-traces of water; $663.3 / 662.1 \mathrm{~m}(4.1)$ traces of $\mathrm{CO}_{2}$. 


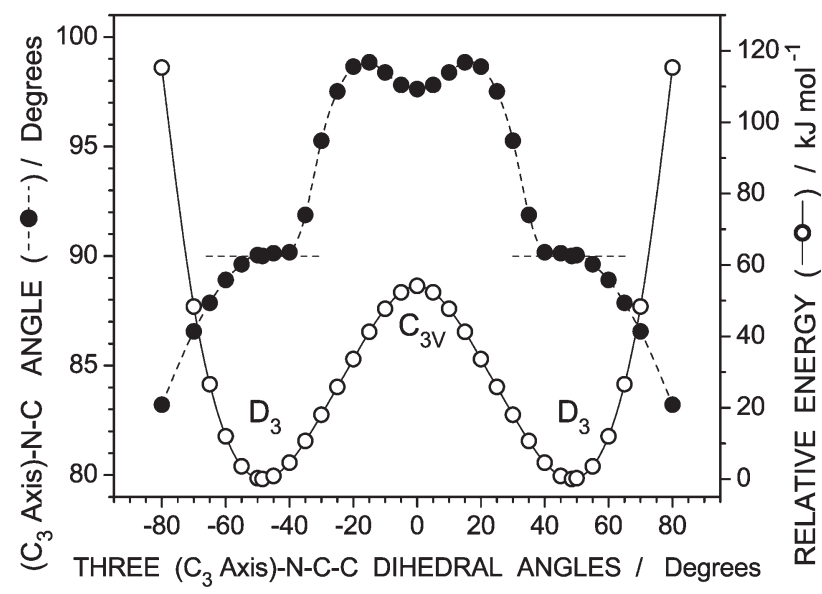

Fig. 3 Relaxed PES scan in the molecule of triphenylamine with the $C_{3}$ symmetry conserved at every point. Calculation at the DFT(B3LYP)/6-31G** level of theory. The $C_{3}$ axis passes through the nitrogen atom and is perpendicular to the plane constituted by the three central carbon atoms (adjacent to the nitrogen). All three propeller blades (phenyl rings) are rotated in the concerted way. The horizontal dashed line with ordinate 90 degrees corresponds to the planar central NCCC fragment. In the minima the molecule belongs to the $D_{3}$ point group and the NCCC moiety adopts a planar structure. In the transition state symmetry changes to $C_{3 \mathrm{v}}$.

subgroups. Hence, to investigate the pathways for intramolecular rearrangements in TPA, working within these symmetry constrains appears to be the logical option. Accordingly, in the calculations described below, three, two or one CNCC dihedral angles were incrementally varied in a concerted way, whereas the remaining geometry parameters were allowed to relax within the limits of one of the above mentioned symmetry constraints.

In the first case, corresponding to the concerted rotation of all three phenyl groups in the same direction, symmetry with respect to the $C_{3}$ axis was conserved throughout the whole conversion. At each of the intermediate points, a chosen value of the $\left(C_{3}\right.$ axis)-N-C-C dihedral angle $\ddagger$ was fixed, while all other parameters were optimized. The potential energy profile for such a concerted torsion is presented in Fig. 3, while the optimized structures along the reaction pathway are shown in the $\mathrm{ESI} \dagger$ as an animation (Fig. S2). In the region where the $\left(C_{3}\right.$ axis)-N-C-C dihedral angle deviates from the equilibrium geometry by $\pm 10^{\circ}$, the deviation from planarity at the central nitrogen atom does not exceed $1^{\circ}$ (Fig. 3), but, at the top of the barrier, the central NCCC atoms are no longer coplanar (the $\left(C_{3}\right.$ axis)-N-C angle is no more equal to $\left.90^{\circ}\right)$. This transition state structure (structure II in Fig. 1), which is higher in energy by $54 \mathrm{~kJ} \mathrm{~mol}^{-1}$ than the energy minima, has $C_{3 \mathrm{v}}$ symmetry. The pyramidalization at the central nitrogen atom exhibited by this structure may be defined by the angle between the $C_{3}$ symmetry axis and a $\mathrm{CN}$ bond, which is equal to $97.6^{\circ}$. Correspondingly, the three $\mathrm{CNC}$ angles are equal to $118.3^{\circ}$. The last value, when compared with the tetrahedral angle $\left(109.47^{\circ}\right)$, shows the relatively small pyramidalization exhibited by TPA in the discussed transition state geometry. Interestingly, the calculated vibrational spectrum for this transition state structure shows three vibrations with imaginary frequencies, demonstrating that it is not a first order saddle point. Hence, there must exist another, lower energy path for the conversion of the left-hand rotating to the right-

$\ddagger$ Note that for geometries with a planar NCCC moiety, like the minimum energy structure, the ( $\mathrm{C} 3$ axis)- $\mathrm{N}-\mathrm{C}-\mathrm{C}$ dihedral angle, corresponding here to the reaction coordinate, is complementary to $90^{\circ}$ with respect to the "pitch of the blade" angle. However, for non-planar arrangements around the central nitrogen atom such relationship is not valid any more.

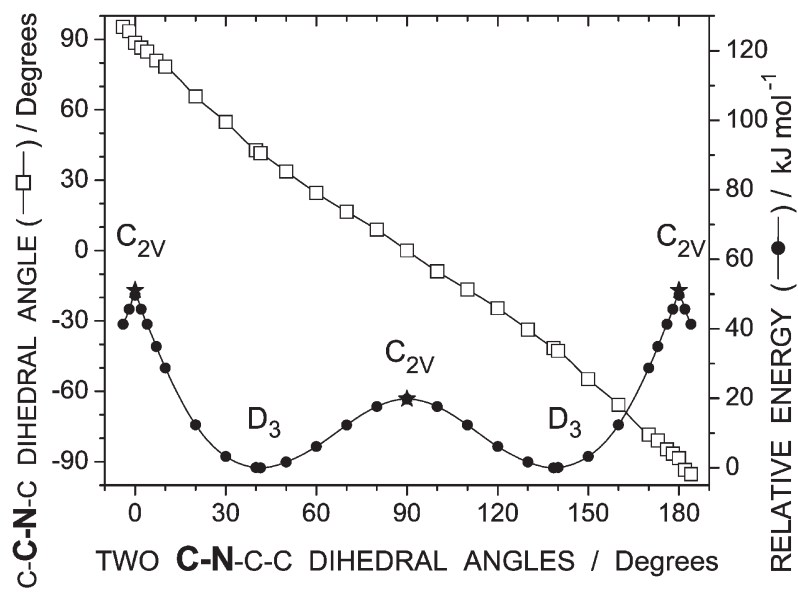

Fig. 4 Relaxed PES scan with the $C_{2}$ point group of symmetry imposed on TPA molecule. Calculation at the DFT(B3LYP)/6$31 \mathrm{G}^{* *}$ level of theory. The $C_{2}$ axis passes through one of the phenyl rings (the "central" blade of the propeller), coincides with one of the $\mathrm{C}-\mathrm{N}$ bonds and is bisecting the opposite $\mathrm{C}-\mathrm{N}-\mathrm{C}$ angle. The position of the $C_{2}$ axis is designated with bold face font and bigger font size in the abscissa and ordinate titles. Note that the ordinate scale for energy is chosen identical to that of Fig. 3. Such a representation demonstrates that both saddle points belonging to the $C_{2 \mathrm{v}}$ point group are lower (or much lower) in energy than the $C_{3 \mathrm{v}}$ one. The points marked with stars designate the saddle points optimized with explicit $C_{2 \mathrm{v}}$ symmetry.

hand rotating form of TPA, which should be associated with a transition state belonging to a different (lower than $C_{3 \mathrm{v}}$ ) symmetry point group. As it could be anticipated, the three imaginary frequencies: $63.5 \mathrm{i} \mathrm{cm}^{-1}\left(\mathrm{~A}_{2}\right.$ symmetry) and $21.2 \mathrm{i} \mathrm{cm} \mathrm{cm}^{-1}$ (doubly degenerated; E symmetry), obtained for the $C_{3 \mathrm{v}}$ structure II correspond to concerted rotations of the phenyl rings around the $\mathrm{C}-\mathrm{N}$ bonds. They rotate either all in phase $\left(\mathrm{A}_{2}\right.$ symmetry) or in different directions (E symmetry). These normal modes indicate the directions of shallow cuts through the potential energy surface of the system.

It is also interesting to point out that the $C_{3 \mathrm{v}}$ structure II resulting from the $C_{3}$ symmetry constrained interconversion between the symmetrically identical minima is located in the conformational space of TPA close to the geometry corresponding to the highest possible symmetry of the molecule: $D_{3 \mathrm{~h}}$. In this $D_{3 \mathrm{~h}}$ geometry, the central NCCC atoms are co-planar and the phenyl rings are perpendicular to the NCCC plane. Geometry optimization with explicit $D_{3 \mathrm{~h}}$ symmetry yields a structure that is $55 \mathrm{~kJ} \mathrm{~mol}^{-1}$ higher in energy than the global energy minimum of the system. Noteworthy, this structure is only $1 \mathrm{~kJ} \mathrm{~mol}^{-1}$ above the $C_{3 \mathrm{v}}$ structure II. The comparison of the simulated spectra of the $D_{3 \mathrm{~h}}$ and $C_{3 \mathrm{v}}$ structures shows that the $D_{3 \mathrm{~h}}$ structure has an additional imaginary frequency at $40.9 \mathrm{i} \mathrm{cm} \mathrm{cm}^{-1}$, belonging to the $\mathrm{A}_{2}{ }^{\prime \prime}$ symmetry species. The vibration corresponding to this frequency is responsible for pyramidalization of the system at the central nitrogen and connects two identical mirror-like $C_{3 \mathrm{v}}$ structures. The small energy difference between the $D_{3 \mathrm{~h}}$ and $C_{3 \mathrm{v}}$ structures demonstrates the flexibility of the system with respect to the pyramidalization.

The second possibility of conversion from the left-hand rotating to the right-hand rotating minimum-energy structures of TPA can be achieved by torsion of two phenyl rings in one direction and the third in the opposite way (Fig. 1, lower row). In this case, the potential energy scan has been performed under $C_{2}$ minimal symmetry constraint. Along the reaction coordinate, two $\mathrm{C}-\mathrm{N}-\mathrm{C}-\mathrm{C}$ torsional angles (determining the pitch of the blade of the two phenyl rings which rotate in the same direction) were incremented in a concerted way, while all the remaining geometric parameters of the molecule were 
optimized. The optimization concerned also the $\mathrm{C}-\mathrm{N}-\mathrm{C}-\mathrm{C}$ torsional angle determining the pitch of the blade of the third ring, whose $\mathrm{C}-\mathrm{N}$ bond coincides with the $C_{2}$ symmetry axis. The imposed symmetry constraint to minimal $C_{2}$ symmetry requires that all points along the reaction coordinate correspond to structures with a planar NCCC moiety. The potential energy profile resulting from this scan is presented in Fig. 4 (see also the animation in Fig. S3 of the ESI $\dagger$ ). At the top of the energy barrier the molecule has $C_{2 \mathrm{v}}$ symmetry (two rings are perpendicular and one is co-planar with respect to the plane defined by the central NCCC atoms; structure III in Fig. 1) and the energy is higher than that of the minimum energy structures by $19.8 \mathrm{~kJ} \mathrm{~mol}^{-1}$, i.e., ca. three times lower than that obtained for the transition structure resulting from the minimal $C_{3}$ symmetry constrained scan. In the vibrational spectrum calculated for the top-of-the-barrier structure only one imaginary frequency (35.1(i) $\mathrm{cm}^{-1}, \mathrm{~A}_{2}$ symmetry) is present. This frequency is associated with the synchronized rotation in the same direction of the two "lateral" phenyl rings around the $\mathrm{N}-\mathrm{C}$ bonds, so that during the vibration the molecule retains the $C_{2}$ symmetry. The presence of only one imaginary frequency shows that the structure III is a first order saddle point, and then it is a true transition state for the interconversion between the two mirror-like minima of TPA. Let us note that the monotonous, almost linear, decrease of the pitch of the central blade (optimized parameter, squares in Fig. 4) with a concomitant increase of the pitch of the two other blades (reaction coordinate) confirms that the central blade rotates along the scan in the direction opposite to the two lateral blades.

Finally, a potential energy scan performed by defining as reaction coordinate the pitch of the blade angle of only one phenyl ring was also undertaken (Fig. 5 and Fig. S4 of the $\mathrm{ESI} \dagger$ ). No symmetry restrictions were applied in this case. As the starting point for the scan (outermost left point on the plot shown in Fig. 5) the geometry of the lowest energy saddle point ( $C_{2 \mathrm{v}}$ point group, structure III) obtained in the " $C_{2}$ scan" was chosen. The optimized energies (solid line) at the points with

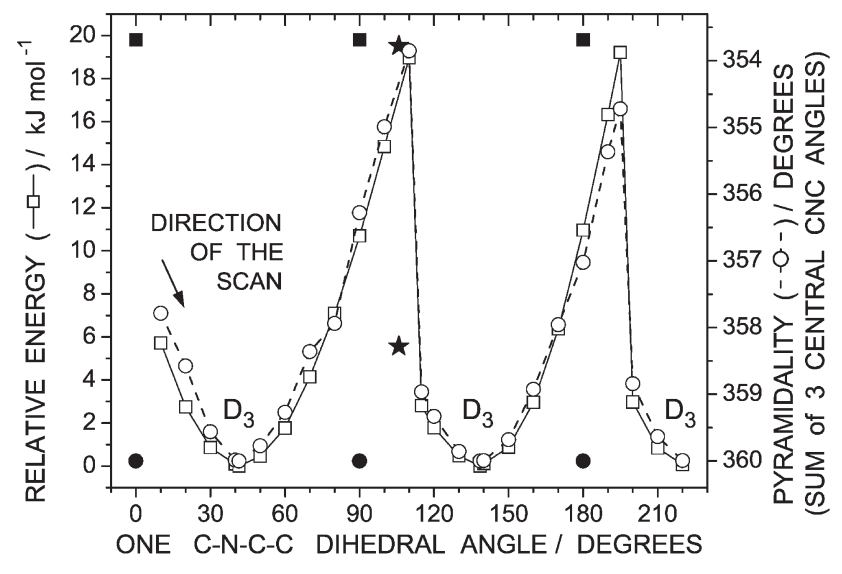

Fig. 5 Relaxed PES scan with no symmetry restrictions $\left(C_{1}\right.$ point group) imposed on the TPA molecule. Calculation at the DFT(B3LYP)/6-31G** level of theory. The pitch of only one blade is optimized. The energies (filled squares) and pyramidalities (filled circles) of the lowest $C_{2 \mathrm{v}}$ saddle points are shown for comparison. Pyramidality of 360 degrees corresponds to the planar NCCC fragment. Saddle points ( $C_{\mathrm{s}}$ symmetry) are designated by stars: upper star: energy, lower star: pyramidality. This saddle point has one propeller blade approximately coplanar with the central NCCC fragment, and the other two are perpendicular (similar to structure III in Fig. 1). For the neighbouring saddle points the "coplanar" propeller blade is not the same. By completing 180 degrees rotation of one of the phenyl rings the system returns to the starting position. During the 180 degrees rotation two propeller blades alternate in adopting the role of the central blade. abscissas equal to $90^{\circ}$ and $180^{\circ}$, obtained without any symmetry constraint, are much lower than those obtained when minimal $C_{2}$ symmetry was imposed (shown as filled squares in the figure). The increase in energy due to rotation of the phenyl ring away from its minimum energy configuration is compensated by pyramidalization of the molecule (dashed line; compare the pyramidalization of the molecule when no symmetry is imposed, for abscissas equal to $90^{\circ}$ and $180^{\circ}$, with that of the $C_{2 \mathrm{v}}$ saddle point, represented in the figure as filled circles). It is also obvious from this plot that the energy of the system is a linear function of the pyramidalization. The smooth energy increase to the threshold of $c a$. $19-20 \mathrm{~kJ} \mathrm{~mol}^{-1}$ (the energy of the lowest $C_{2 \mathrm{v}}$ saddle point) was accompanied by an increase of pyramidalization at the central nitrogen atom. Then, a jump in the pyramidalization occurs (the nitrogen atom passes to the opposite side of the plane formed by the three central carbon atoms) and, concomitantly, a big decrease in the energy ( $c a .15 \mathrm{~kJ} \mathrm{~mol}^{-1}$ ) occurs. This behaviour justifies the dependence of the shape of the potential energy profile on the direction of the scan as well as the fact that energy is not a smooth function of the driving coordinate. The saddle point in this scan belongs to the $C_{\mathrm{s}}$ point group. In this structure, the central NCCC moiety is not planar and, interestingly, the energy (with account for the zero point vibrational energy) is equal to that of the $C_{2 \mathrm{v}}$ saddle point previously discussed. As in the case of the $C_{2 \mathrm{v}}$ saddle point, the $C_{\mathrm{s}}$ transition state structure has only one imaginary frequency $\left(35.6(\mathrm{i}) \mathrm{cm}^{-1}, \mathrm{~A}^{\prime \prime}\right.$ symmetry). This frequency is related to the concerted rotation of the two lateral phenyl rings around $\mathrm{N}-\mathrm{C}$ bonds in one direction while the central phenyl ring rotates in the opposite direction.

The energy of the transition state corresponding to the top of the barrier separating the left-hand rotating and right-hand rotating forms of TPA obtained in the scan with no symmetry constraints is almost the same as that calculated in the scan with the conservation of the $C_{2}$ axis. Also the nature of normal vibrations corresponding to the unique imaginary frequencies in these two scans is almost identical. Analysis of the optimized geometries along the symmetry unconstrained scan leads to the important conclusion that along this scan there is always one phenyl ring which rotates into the opposite direction with respect to the other two phenyl rings. Virtually, this and the $C_{2}$ scans are very similar; they differ only by giving the molecule the possibility (or not) of pyramidalization at the central nitrogen atom. Indeed, both scans can be schematically represented by the lower row in Fig. 1.

The drastic difference between the heights of the energy barriers $\left(54\right.$ and $20 \mathrm{~kJ} \mathrm{~mol}^{-1}$ ) for the transformation between the two TPA minima following the two paths presented in Fig. 1 strongly suggests that the second route should be preferred. On the other hand, both the relatively high $\left(20 \mathrm{~kJ} \mathrm{~mol}^{-1}\right)$ energy barrier for interconversion between the two equivalent by symmetry minima and the very large amplitude movement necessary for this conversion are consistent with a low probability of barrier crossing. This may justify the absence of spectral line doubling, due to existence of two identical minima, in the high resolution LIF excitation spectra of TPA in a supersonic jet expansion. ${ }^{25}$

\section{Conclusion}

The experimental FTIR spectrum of TPA monomers isolated in a low temperature argon matrix is reported for the first time. Theoretical simulations predict the equilibrium geometry of the molecule as being $D_{3}$ symmetry, propeller like, with a pitch of blade angle of $c a .41 .5^{\circ}$ and a planar NCCC moiety. A good agreement between the experimental and theoretically calculated spectra allowed a positive assignment of the observed infrared bands and indicates that the theoretically 
optimized geometry of TPA closely matches that of the matrix isolated monomer of the compound. The detailed investigation of the possible pathways connecting the two mirror-like minima of TPA enabled the identification of several transition states belonging to different symmetry point groups and clearly revealed that the interconversion between the minima shall preferentially occur through a channel where two phenyl groups rotate in one direction while the third one rotates in the opposite direction. The study of the dynamics of intramolecular rearrangements in TPA revealed also a strong cross-correlation between the pitch of blade angle and pyramidalization in determining the energetically preferred structures of TPA.

\section{Acknowledgements}

IR and RF acknowledge the financial support from Fundação para a Ciência e a Tecnologia, Lisbon (grant FCT \#SFRH/ $\mathrm{BPD} / 1661 / 2000$ and research project POCTI/43366/QUI/ 2001). NC thanks Department of Science and Technology and Council of Scientific and Industrial Research, Govt. of India, for the financial support.

\section{References}

1 W. L. Yu, J. Pei, W. Huang and A. J. Heeger, Chem. Commun., 2000, 681-682.

2 J. P. Chen, H. Tanabe, X. C. Li, T. Thoms, Y. Okamura and K. Ueno, Synth. Met., 2003, 132, 173-176.

3 F. L. Bai, M. Zheng, G. Yu and D. B. Zhu, Thin Solid Films, 2000, 363, 118-121.

4 D. Troadec, G. Veriot and A. Moliton, Synth. Met., 2002, 127, $165-168$.

5 T. Noda and Y. Shirota, J. Lumin., 2000, 87-89, 1168-1170.

6 C. S. Ha, J. H. Shin, H. Lim and W. J. Cho, Mater. Sci. Eng. B, 2001, 85, 195-198.

7 R. A. Holroyd, J. M. Preses, E. H. Boettcher and W. F. Schmidt, J. Phys. Chem., 1984, 88, 744-749.

8 J. Ostrauskaite, H. R. Karickal, A. Leopold, D. Haarer and M. Thelakkat, J. Mater. Chem., 2002, 12, 58-64.

9 Y. Yasuda, T. Kamiyama and Y. Shirota, Electrochim. Acta, 2000, 45, 1537-1541.

10 J. Steiger, R. Schmechel and H. von Seggern, Synth. Met., 2002, 129, 1-7.

11 W. Holzer, A. Penzkofer, H. H. Horhold, D. Raabe and M. Helbig, Opt. Mater., 2000, 15, 225-235.

12 W. Holzer, A. Penzkofer, H. Tillmann, E. Klemm and H. H. Horhold, Synth. Met., 2001, 124, 455-465.

13 Y. Nishikata, S.-I. Fukui, M.-A. Kakimoto, Y. Imai, K. Nishiyama and M. Fujihira, Thin Solid Films, 1992, 210-211, 296-298.
14 Y. Nishikata, S. I. Fukui, M. A. Kakimoto, Y. Imai, K. Nishiyama and M. Fujihira, Mol. Cryst. Liq. Cryst., 1993, 224, 95-109.

15 B. P. Chandra, Indian J. Pure Appl. Phys., 1980, 18, 743-746.

16 B. P. Chandra and J. I. Zink, J. Phys. Chem., 1982, 86, 4138-4141.

17 M. Lor, J. Thielemans, L. Viaene, M. Cotlet, J. Hofkens, T. Weil, C. Hampel, K. Muellen, J. W. Verhoeven, M. Van der Auweraer and F. C. De Schryver, J. Am. Chem. Soc., 2002, 124, 9918-9925.

18 M. Thelakkat, Macromol. Mat. Eng., 2002, 287, 442-461.

19 N. Chattopadhyay, C. Serpa, L. G. Arnaut and S. J. Formosinho, Phys. Chem. Chem. Phys., 2001, 3, 3690-3695.

20 C. B. Duke, J. W.-.P. Lin, A. Paton, W. R. Salaneck and K. L. Yip, Chem. Phys. Lett., 1979, 61, 402-406.

21 M. Malagoli and J. L. Brédas, Chem. Phys. Lett., 2000, 327, 13-17.

22 J. Pacansky, R. J. Waltman and H. Seki, Bull. Chem. Soc. Jpn., 1997, 70, 55-59.

23 C. Kvarnstrom, A. Petr, P. Damlin, T. Lindfors, A. Ivaska and L. Dunsch, J. Solid State Electrochem., 2002, 6, 505-512.

24 H. B. Lueck, J. L. McHale and W. D. Edwards, J. Am. Chem. Soc., 1992, 114, 2342-2348.

25 G. Meijer, G. Berden, W. L. Meerts, H. E. Hunziker, M. S. de Vries and H. R. Wendt, Chem. Phys., 1992, 163, 209-222.

26 A. N. Rodionov, N. I. Ruch'eva, K. L. Rogozhin and D. N. Shigorin, Zh. Prikl. Spektrosk., 1974, 20, 534-535.

27 A. N. Sobolev, V. K. Belsky, I. P. Romm, N. Y. Chernikova and E. N. Guryanova, Acta Crystallogr. Sect. C, 1985, C41, 967-971.

28 P. Knobloch and M. Stockhausen, Angew. Chem., Int. Ed. Engl., 1964, 3, 230-231.

29 I. Janic and M. Kakas, J. Molecular Structure, 1984, 114, $249-252$.

30 R. D. W. Kemmitt, R. H. Nuttall and D. W. A. Sharp, J. Chem. Soc., 1960, 46-50.

31 C. W. N. Cumper and A. P. Thurston, J. Chem. Soc. B, 1971, $422-426$.

32 A. D. Becke, Phys. Rev. A, 1988, 38, 3098-3100.

33 C. T. Lee, W. T. Yang and R. G. Parr, Phys. Rev. B, 1988, 37, 785-789.

34 S. H. Vosko, L. Wilk and M. Nusair, Can. J. Phys., 1980, 58, 1200-1211.

35 M. J. Frisch, G. W. Trucks, H. B. Schlegel, G. E. Scuseria, M. A. Robb, J. R. Cheeseman, V. G. Zakrzewski, J. A. Montgomery, Jr., R. E. Stratmann, J. C. Burant, S. Dapprich, J. M. Millam, A. D. Daniels, K. N. Kudin, M. C. Strain, O. Farkas, J. Tomasi, V. Barone, M. Cossi, R. Cammi, B. Mennucci, C. Pomelli, C. Adamo, S. Clifford, J. Ochterski, G. A. Petersson, P. Y. Ayala, Q. Cui, K. Morokuma, D. K. Malick, A. D. Rabuck, K. Raghavachari, J. B. Foresman, J. Cioslowski, J. V. Ortiz, A. G. Baboul, B. B. Stefanov, G. Liu, A. Liashenko, P. Piskorz, I. Komaromi, R. Gomperts, R. L. Martin, D. J. Fox, T. Keith, M. A. Al-Laham, C. Y. Peng, A. Nanayakkara, M. Challacombe, P. M. W. Gill, B. Johnson, W. Chen, M. W. Wong, J. L. Andres, C. Gonzalez, M. Head-Gordon, E. S. Replogle and J. A. Pople, Gaussian 98, Revision A.9, Pittsburgh PA, 1998.

36 D. H. Whiffen, J. Chem. Soc., 1956, 1350-1356.

37 T. Stepanenko, L. Lapinski, M. J. Nowak and L. Adamowicz, Vib. Spectrosc., 2001, 26, 65-82. 